\title{
Stability of the adaptive-optic point spread function: metrics, deconvolution, and initial Palomar results
}

Eric E. Bloemhof, Kenneth Alan Marsh, Richard G. Dekany, Mitchell Troy, J. Marshall, et al.

Eric E. Bloemhof, Kenneth Alan Marsh, Richard G. Dekany, Mitchell Troy, J. Marshall, Ben R. Oppenheimer, Thomas L. Hayward, Bernhard Rainer Brandl, "Stability of the adaptive-optic point spread function: metrics, deconvolution, and initial Palomar results," Proc. SPIE 4007, Adaptive Optical Systems Technology, (7 July 2000); doi: 10.1117/12.390384 


\title{
Stability of the Adaptive-Optic Point-Spread Function: Metrics, Deconvolution, and Initial Palomar Results
}

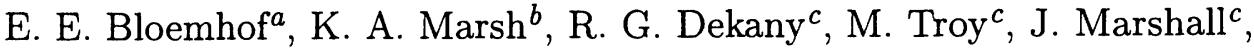 \\ B. R. Oppenheimer ${ }^{d}$, T. L. Hayward ${ }^{e, f}$, and B. Brandl ${ }^{e}$ \\ ${ }^{a}$ Palomar Observatory, California Institute of Technology, Pasadena, CA 91125 USA \\ ${ }^{b}$ IPAC, California Institute of Technology, Pasadena, CA 91125 USA \\ ${ }^{c}$ Jet Propulsion Lab, California Institute of Technology, Pasadena, CA 91109 USA \\ ${ }^{d}$ Astronomy Department, University of California, Berkeley, CA 94620 USA \\ ${ }^{e}$ Astronomy Department, Cornell University, Ithaca, NY 14853

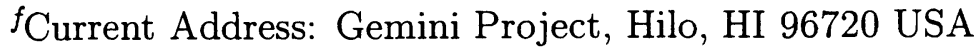

\begin{abstract}
The essential benefit of adaptive optics is delivering a telescope point-spread function (PSF) limited by diffraction rather than by atmospheric turbulence. In practice, achieving diffraction-limited PSF diameters is relatively routine for modern high-order systems, at least within a restricted isoplanatic patch containing the guide star. The lowerintensity wings of the PSF, though, are often highly complex in their structure and subject to variability over short time scales. Spurious bright knots can occur among the secondary Airy maxima, and the "waffle-mode" artifact may be problematic with a broad class of adaptive optics approaches. Good temporal stability of the adaptive-optic PSF is generally highly desirable if the full advantage of that spectacular PSF is to be realized; it is absolutely critical for many specific high-resolution programs that can only be attempted with adaptive optics. In the course of commissioning the high-order adaptive optics system built at JPL for the Palomar 200-inch telescope, we have investigated PSF stability in the field under a variety of conditions. We discuss here our experimental findings at Palomar, and their implications for some key scientific programs.
\end{abstract}

Keywords: adaptive optics, point-spread function, deconvolution

\section{INTRODUCTION}

PALAO, the Palomar adaptive optics system for the 200-inch telescope, is based on a 349-actuator PMN deformable mirror and a visible-light Shack-Hartmann wavefront sensor that places a 16x16 array of subapertures across the pupil. Centroids are measured by a low-noise $64 \times 64 \mathrm{CCD}$ capable of readout rates as high as $500 \mathrm{~Hz}$. Overall tip-tilt is corrected by a separate fast-steering mirror, with a closed-loop bandwidth of $\sim 5 \mathrm{~Hz}$. A comprehensive description of the instrument's current status may be found elsewhere in this volume, ${ }^{1}$ and some aspects of its design and construction have also been published. ${ }^{2-4}$ PALAO feeds a specially-designed near-infrared (JHK) science camera called PHARO (Palomar High Angular Resolution Observer). ${ }^{5}$ Although designed with sufficient flexibility to accommodate a laser beacon, PALAO is now and for the foreseeable future a natural guide star system. PALAO first achieved high-order correction at the telescope in December, 1998, and has been used productively during the 1999 observing season for a variety of scientific projects. Initial results have been most encouraging, and are discussed elsewhere in this volume. ${ }^{6,7}$

The near-diffraction-limited PSF delivered by PALAO or any other adaptive optics system is subject to variation with seeing conditions and, to a lesser extent, with flexure and optical alignment of the adaptive optics system itself. Seeing conditions at Palomar can undergo dramatic qualitative changes on hourly timescales, and significant quantitative changes more quickly still; these changes are evident in real time on the wavefront-sensor display, for example. Stability of the PSF is a critical requirement for most scientific projects. In this paper, we aim to characterize PSF stability with data taken during the inaugural observing season of PALAO. In the course of these investigations, we propose some new PSF metrics that go beyond the quantities traditionally used (i.e. the width of

Further author information: (Send correspondence to E.E.B.)

E.E.B.: E-mail: eeb@astro.caltech.edu; WWW: http://astro.caltech.edu/ eeb; Telephone: 6263953658 ; Fax: 626 568 1517

In Adaptive Optical Systems Technology, Peter L. Wizinowich, Editor,

Proceedings of SPIE Vol. 4007 (2000) $\bullet 0277-786$ X/00/ $\$ 15.00$ 
the central peak, describing gross achievement of the diffraction limit, and the Strehl ratio, essentially describing the total amount of light dispersed into a low-intensity surrounding halo). These new metrics are intended to quantify some details of the PSF, particularly relating to the structure of the halo, that are not addressed by the coarser traditional metrics but that nonetheless play an important role in assessing the performance of an adaptive optics system and its suitability for some of its most important scientific applications. The temporal PSF behavior reported in this paper will deal with the on-axis case: anisoplanaticity studies, detailing the spatial variation of PSF quality, particularly as a function of distance from the guide star, will be reported elsewhere in this volume. ${ }^{8}$

\section{THE NATURE OF THE PROBLEM: THE IDEAL DIFFRACTION-LIMITED TELESCOPE PSF VS. THE PRACTICAL ADAPTIVE-OPTIC PSF}

Our experience with PALAO confirms that the quality of the adaptive correction is a strong function of the initial, uncorrected seeing. In Figure 1 we plot the theoretical diffraction-limited PSF for a $5 \mathrm{~m}$ telescope (left) next to a fairly representative PALAO PSF (right) obtained under conditions of relatively good seeing and adaptive correction. The theoretical pattern includes the effects of obscuration by the secondary mirror (more precisely, by the secondary block in the science camera's pupil mask), whose $\sim 36 \%$ fractional diameter as seen from the $\mathrm{f} / 15.7$ Cassegrain focus causes a $\sim 7 \%$ narrowing of the PSF's main lobe and a substantial increase in the height of the first "Airy" ring (from less than $2 \%$ of the main peak to roughly $6 \%$ ).
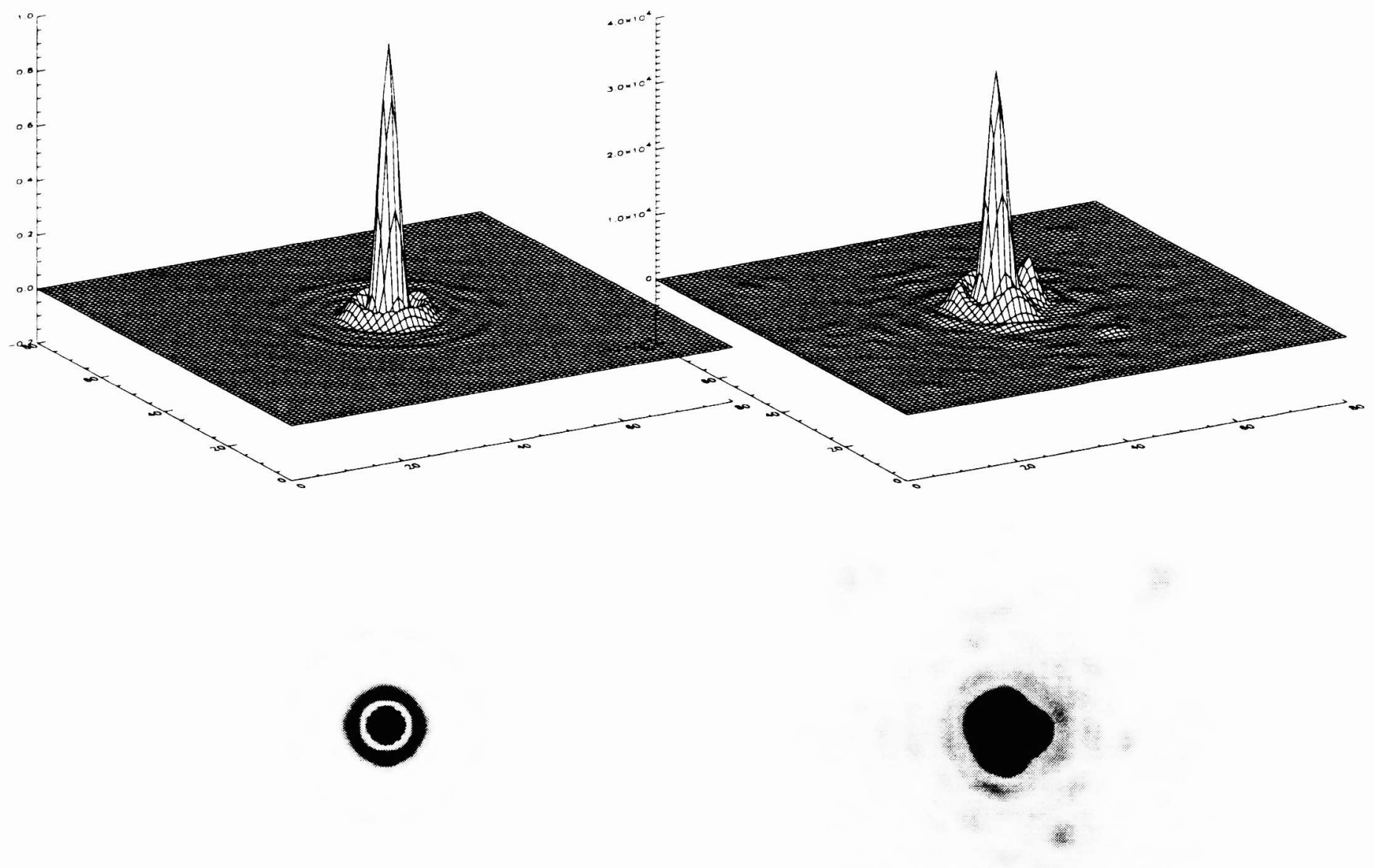

Figure 1. (Left plots) Ideal diffraction-limited PSF for a $5 \mathrm{~m}$ telescope at $\lambda=2.2 \mu \mathrm{m}$, with a central obscuration equal to $36 \%$ of the primary diameter; (Right plots) Experimental PALAO PSF at $2.2 \mu \mathrm{m}$, in relatively good seeing. In each case, pixels are 25 mas, and a \pm 1.00 arc second ( \pm 40 pixel) region on each side of the peak is plotted. The grayscale plots (bottom) use a logarithmic intensity scaling to emphasize the low-level structure in the halo. 
Figure 1 (right) illustrates several features typical of a good-quality PSF delivered by a high-order adaptive optics system. The shape of the main peak is excellent, and it has a scale only slightly larger than that of the diffractionlimited ideal, about 89 milliarcseconds (mas) FWHM at this wavelength $(2.2 \mu \mathrm{m})$. This is of course more than an order of magnitude improvement over the linear size of the initial arc-second seeing disk.

There are also clear signatures corresponding to the Airy rings, whose amplitudes agree well with the ideal case. These rings are strongly modulated, though, by bright knots of light. One expects a four-fold symmetric pattern of modulation to be generated by the spiders of the telescope (again, more precisely, by their slightly-oversized pupil-plane mask), and this effect is included in the diffraction calculation of Figure 1 (left). However, the resulting modulation of the Airy rings is far smaller than in the experimentally-observed case. Furthermore, the symmetry of the observed modulation on the first Airy ring appears possibly threefold, rather than clearly fourfold; more will be said about this in $\S 3$, where we will study its temporal behavior.

Diffraction from the spider mask will also produce a four-fold-symmetric pattern of light extending in the four cardinal directions from the peak, and there is some suggestion of such a pattern in Figure 1 (right); again, though, spider diffraction (Figure 1 left) appears insufficiently bright to account for the observed pattern. Also present in the low-level structure of the experimental PSF of Figure 1 (right) is at least one localized artifact due to "ghosting" in the optics of the science camera. Ghosts are cosmetically irritating but not too problematic in a practical sense, owing to their constancy. The most prominent example is the knot below and to the right of the main peak in Figure 1 (right), about halfway to the boundary of the plot. This is the brightest artifact of any kind so distant (about 1 arcsecond) from the center, with a surface brightness roughly $2 \%$ of that of the main peak.

Another imperfection unique to the adaptive-optic PSF is made more visible in the logarithmic grayscale plot of Figure 1 (lower right), which suppresses the bright peak and enhances low-level emission. In addition to the quasi-random arrangement of bright knots in the halo, there are four spurious peaks arranged in a sqare pattern centered on the source. These are the "waffle-mode" artifacts characteristic of adaptive optics systems; they tend to creep into images when the high-order lock has been closed for a long time.

Intermingled with these faint diffraction spots and artifacts in the outlying "halo", far from the main peak of Figure 1 (right), are remnant speckles due to imperfectly corrected atmospheric wavefront aberrations. Though low in surface brightness, the total energy content of the halo can be large. For the example PSF of Figure 1, the halo is of order an arcsecond in diameter, and the brighter speckles in this 0.5 -second exposure have a surface brightness roughly a few tenths of $1 \%$ of that of the peak (in longer exposures, the halo is smoother and fainter). Halo speckles may be expected to change over relatively short time scales, while spots of light due to diffraction or other instrumental effects may persist over longer timescales. Since the stray light in the halo from seeing speckles, diffraction, and artifacts must come from the main peak, that peak is substantially lower than in the ideal diffractionlimited case; the plots in Figure 1 are scaled to equal peak intensity, however. These various imperfections in the corrected PSF are clearly problematic for imaging faint companions or circumstellar dust in close proximity to bright stars, and for almost every other application of adaptive optics; it is important to determine the temporal behavior of the extraneous structure of the PSF to devise appropriate observing strategies.

All of the PALAO images presented in this paper were reduced by the standard techniques of astronomical near-infrared array imaging. Dead pixels were detected in flat-field exposures, usually taken on the sky at sunrise or sunset (or, if sky flats were not taken, on an illuminated region of the dome); hot pixels were detected in dark exposures; these bad pixels were removed by an iterative algorithm that replaces them with an average of contiguous good pixels. For good pixels, dark exposures provide pixel offsets, and flats, with exposure parameters chosen to match their corresponding observations, provide pixel responsivity calibration. Sky exposures on a nominally empty field allow subtracting out the uniform component of the infrared emission of the sky, or the telescope is dithered between science images to accomplish an equivalent sky measurement (and avoid bad regions on the CCD). Data reduction was greatly aided by a standard package of IDL routines called "AOred", written by Jason Marshall and Mitchell Troy of JPL, and made available to the community of PALAO users.

\section{A QUALITATIVE LOOK AT STABILITY OF THE PSF CORE}

We will define the "core" to include the main peak and the first Airy ring, and will focus primarily on the structure in the Airy ring of the adaptively-corrected PSF reported in the previous section. The shape of the main peak is generally of high quality, as expected from diffraction theory, and deviations there would be on a very fine scale, important mainly if super-resolution beyond the diffraction limit were pursued by deconvolution. 
Each row of Figure 2 shows a quick series of three PSF measurements displayed as contour plots, with levels chosen to emphasize the intermediate spatial scale corresponding to the structure of the first Airy ring. Sets of three images (rows) were separated by about eight minutes of time.
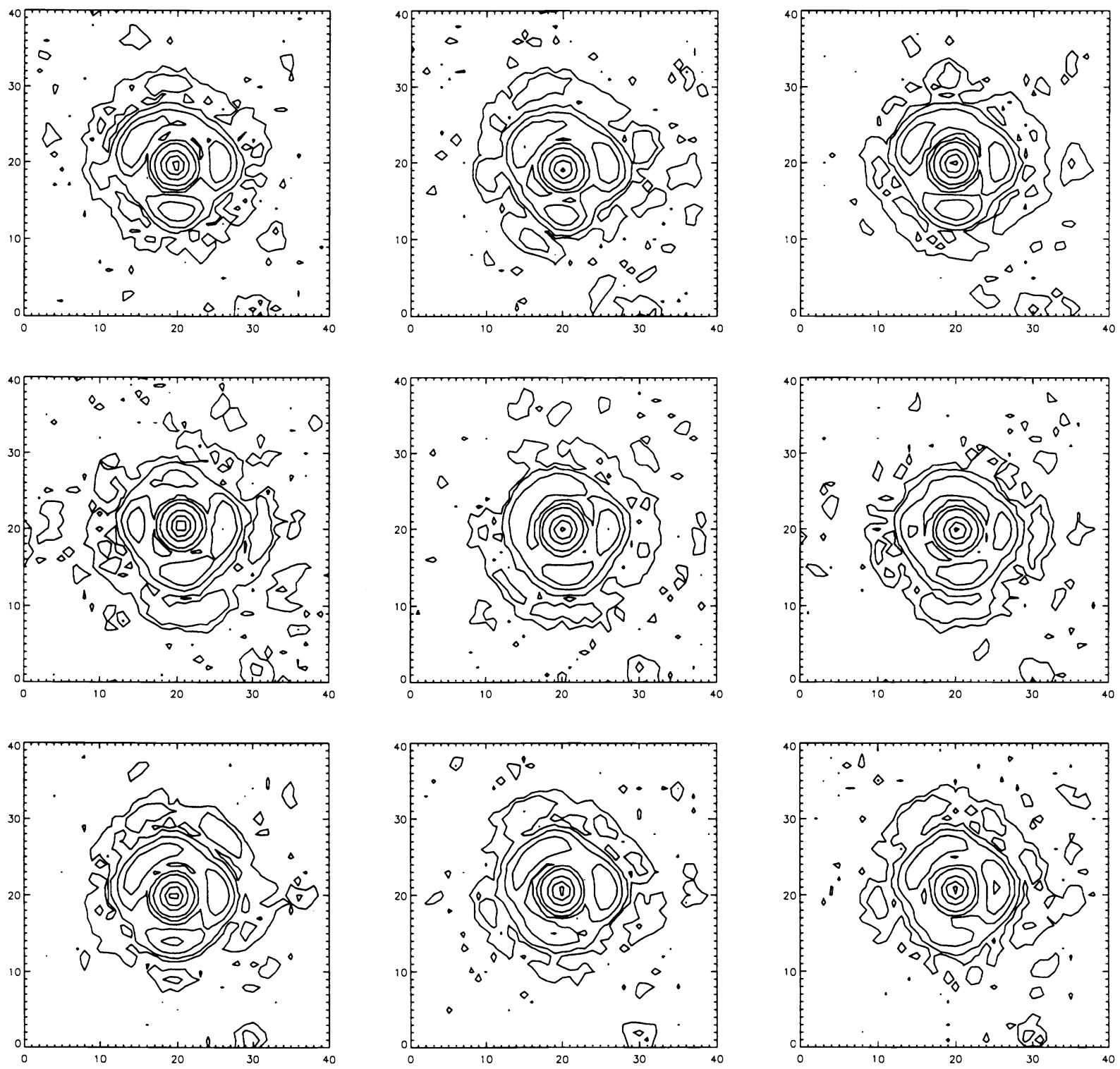

Figure 2. Close-up of the central portions of 0.5-second-exposure PALAO PSFs, clarifying the structure of the first "Airy" ring. In each case, the field of view measures 1.0 arcseconds on a side (note $\times 2$ scale change from Figure 1 ). Contours are peak intensity divided by $1.05,1.33,2,5,10,20,50$, and 100 . The top row has three images taken in quick succession at $t=0,4.4$, and 8.2 seconds. The middle and bottom rows are also sets of three images taken about 4 seconds apart, roughly 7 minutes and 16 minutes later, respectively, than the top row.

The morphology of the Airy ring is grossly persistent within each set of three images, but the character of the images shifts irregularly back and forth between 3-fold and 4-fold symmetry on timescales of 10 minutes or so, over the 53-minute duration of this particular study (a run of 4-fold symmetry in the last two or three image triplets hints at an evolution, but may be purely coincidental). 
Other stellar images taken on a different night show similar arrangements of bright knots on the first Airy ring. These structures typically have three-fold symmetry, and persist over time scales (many seconds or minutes) much longer than those associated with atmospheric effects.

In seeking an instrumental cause, it is tempting to consider the threefold-symmetric structure seen in infrared PSFs at Palomar, which may even show bright knots on the first Airy ring (K. A. Marsh, private communication). These patterns are thought to be inherited from the threefold-symmetric defining points of the mirror support structure, but any print-through to the front surface of the primary should be smooth enough to be compensated by PALAO's deformable mirror.

More likely is the gradual accumulation of errors in the "centroid offsets", the set of static positions to which individual wavefront-sensor subaperture spots are driven by the closed-loop action of the deformable mirror. These offsets, which correct for known static aberrations in the transfer optics of PALAO and then provide the wavefront template to which the system attempts to adhere in the presence of seeing, are determined in a rather subjective calibration procedure. A fiducial white-light source is imaged on the science camera, and image quality is judged by eye as various pupil phase functions are applied. (This procedure will eventually be automated, through the use of a curvature or phase diversity wavefront sensing algorithm, to determine the aberration content of the wavefront reaching the science camera in a single step).

The centroid offsets, in particular, preserve the calibrated memory of differences between the visible-light optical path to the wavefront sensor, and the infrared path to the science camera. If flexure caused non-common-path differences to creep in, the centroid offsets determined in an earlier calibration step would no longer be appropriate for delivering a perfectly corrected wavefront at the science camera.

\section{A QUALITATIVE LOOK AT STABILITY OF THE PSF HALO}

To examine the time evolution of the PSF halo, we use a data set for which the $1024 \times 1024$ science chip was clocked in a $128 \times 128$ subarrayed mode, expediting the readout and reducing the time between successive images. By this expedient, exposure times are reduced to about 0.2 seconds. A series of 8 consecutive $2.2-\mu \mathrm{m}$ images, typically separated by 2.5 seconds, is presented in Figure 3.
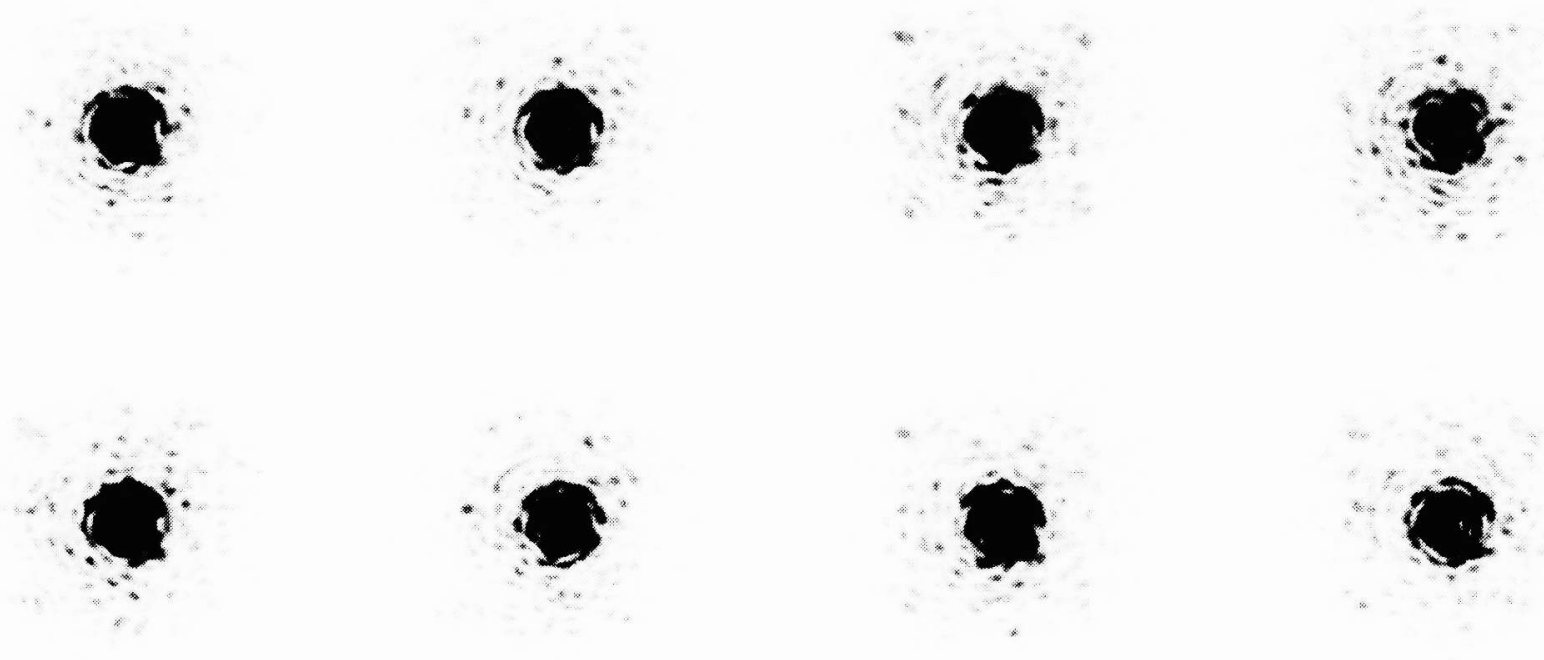

Figure 3. Series of PALAO PSFs taken in rapid succession at $2.2 \mu \mathrm{m}$ in relatively good seeing; a logarithmic display emphasizes low-level structure in the halo. Total time span is about 18 seconds; individual exposures are 0.2 seconds. In each case, the field of view measures 3.2 arcseconds on a side. 
The structure of the halo clearly changes on short time scales, as it must. The canonical atmospheric coherence time at $2.2 \mu \mathrm{m}$ is a little shorter than our exposure time, 0.2 seconds, and much shorter than the time between images. So Figure 3 shows individual speckles rather than a uniform haze in the halo, but those speckles do not persist from one exposure to the next.

\section{STABILITY OF FWHM AND STREHL METRICS OVER AN HOUR}

Two simple metrics that are commonly used to characterize the PSF are FWHM (full-width at half-maximum of the main peak of the PSF) and Strehl ratio (ratio of the peak intensity of the main peak to that expected for a perfect diffraction-limited PSF). FWHM is a basic check on the integrity of the adaptive correction. Roughly speaking, the degree to which the Strehl ratio falls below $100 \%$ measures the diversion of light from the main peak into undesirable extended structure. These metrics are relatively easily calculated, they summarize complex image behavior simply and quantitatively, with single parameters, and they are naturally appropriate to the characteristic core-halo structure of the adaptive-optic PSF.

The science camera in PALAO operates in the near-infrared $\mathrm{J} / \mathrm{H} / \mathrm{K}$ bands $(1.25 / 1.65 / 2.2 \mu \mathrm{m})$, for which the theoretical diffraction-limited PSFs on the Palomar $5 \mathrm{~m}$ telescope (assuming our standard pupil mask equivalent to a $4.88 \mathrm{~m}$ aperture) have FWHM $\sim 50 / 67 / 89$ mas. Under conditions of decent seeing (say, 1 arcsecond at K), corrected PSFs with FWHM quite close to the diffraction limit are obtained at all three near-infrared design wavelengths. As seeing worsens, the FWHM of PALAO's PSF remains close to the diffraction-limited value while the Strehl ratio drops substantially, to a few percent or less. There is then a regime of quite poor seeing, in the neighborhood of 2 arcseconds at $\mathrm{K}$, in which adaptive optics cannot deliver a diffraction-limited FWHM, but can improve the FWHM by factors of 3 or so. As the seeing worsens beyond that point, the ability of PALAO to maintain a lock drops off rapidly.

In Figure 4 we present a long-term ( $~ 53$ minute) study of the traditional metrics, FWHM and Strehl ratio, under good conditions and with relatively good time resolution over short intervals. The data set was discussed in $\S 3$; six groups of three images taken in quick succession (about 4 seconds apart) were obtained roughly every 10 minutes, to span a total of somewhat less than an hour.
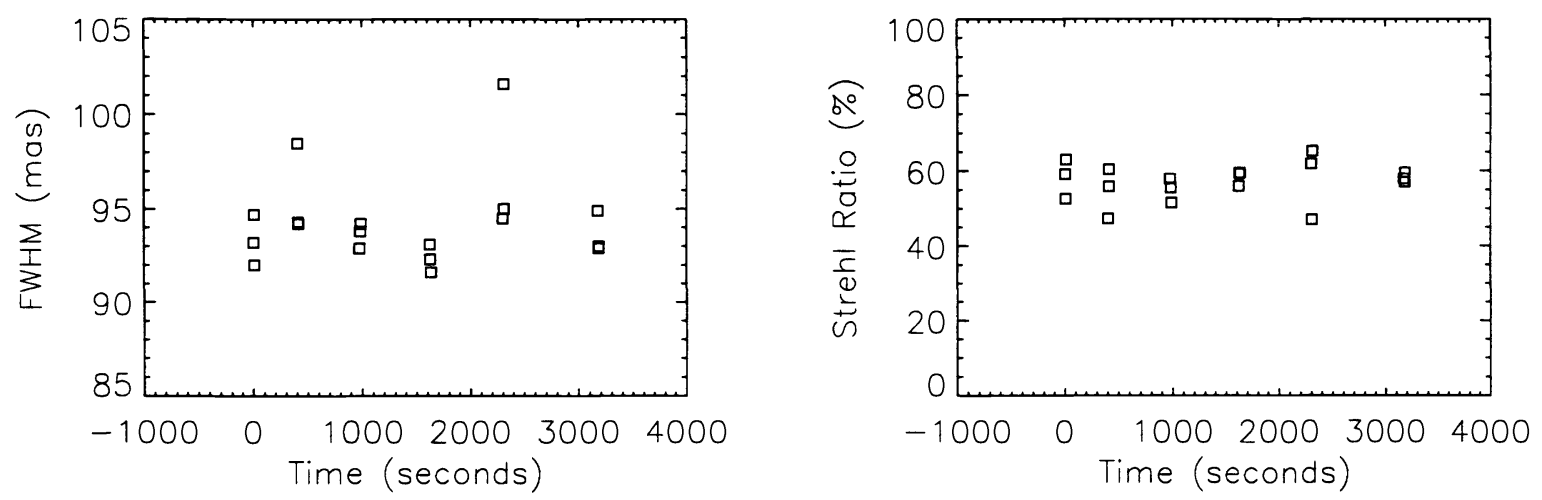

Figure 4. Time evolution of PSF FWHM and Strehl ratio for a series of short-exposure $(0.5 \mathrm{~s}) 2.2-.\mu \mathrm{m}$ images a of point source, gathered in 6 groups of 3 quick images. Total time interval spanned is about 53 minutes. Adaptive optic control loop gains were left constant, and a single star $\left(\mathrm{V} \sim 3.7^{m}\right)$ was tracked at low airmass near the zenith.

Empirically, there is a generally a striking anticorrelation between FWHM and Strehl, which can be seen to some extent in Figure 4. This trend is not surprising, since the FWHM and Strehl ratio are figures of merit describing closely related things: diffraction theory requires that broadening of the PSF's main peak and loss of image power from that peak occur together. For the data of Figure 4, the correlation coefficient is $r \sim-0.4$, formally indicating correlation at the $1.5-\sigma$ level. 
The long-term stability of the adaptive optics system and of the ambient atmospheric conditions is fairly good over the duration of Figure 4, which is representative of typical astronomical integration times. However, dramatic fluctuations within the 8-second span of individual image sets are also evident. This time series illustrates the value of a fast shutter, as planned for the PALAO/PHARO system, to interrupt exposure on the science object in real time when the wavefront sensor detects poor seeing. Excluding images based on sub-threshold values of either of these metrics would save corruption of the high-quality data.

\section{STABILITY OF FWHM AND STREHL OVER SECONDS}

In Figure 5 we present the traditional metrics for the data set of $\S 4$, obtained in a subarrayed readout mode permitting rapid exposures and short gaps between images. The wavelength of observation is $2.2 \mu \mathrm{m} ; 13$ images were obtained in roughly half a minute, for an average time resolution of about 2.5 seconds.

It is noteworthy that in these generally poorer conditions, the dispersion in the metrics is significantly greater. FWHM and Strehl are again anticorrelated, but the correlation is substantially stronger than with the higher-quality (higher-Strehl) data set of $\S 5$ and Figure 4, possibly because of the longer time span in that case: the correlation coefficient is now $r \sim-0.9$, formally indicating correlation at the $3-\sigma$ level.
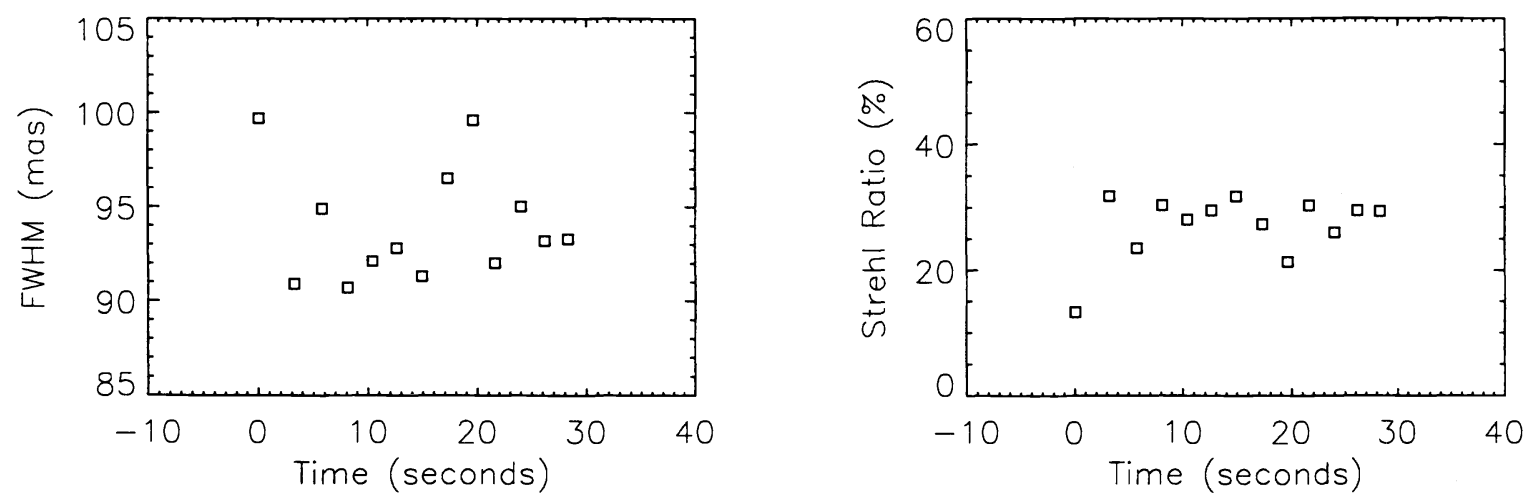

Figure 5. Time evolution of PSF FWHM and Strehl ratio for a series of 13 very-short-exposure $(0.2 \mathrm{~s}$ ) $2.2-\mu \mathrm{m}$ images of a point source. Total time interval is about 30 seconds. Adaptive optic control loop gains were left constant, and a single star $\left(\mathrm{V} \sim 4.1^{m}\right)$ was tracked at low airmass near the zenith.

\section{NEW METRICS: DECONVOLUTION BASED}

The metrics discussed so far primarily describe the main lobe of the PSF: how broad it is (FWHM), and how much flux has it lost to the surrounding halo (Strehl ratio). There is no reason to suppose that there should be a single "best" PSF metric appropriate to all situations; rather, different metrics quantifying different PSF properties may be important, depending on the observational goals.

Stability of the PSF is critical to application of deconvolution algorithms for post-processing of adaptive optic data. Conversely, a number of metrics based on deconvolution may be devised that compare two PSFs at different times to each other or to a third fiducial PSF, either theoretical or measured. In the ideal case, any PSF deconvolved with another should yield a delta function, or a numerical approximation that depends on the number of cycles of the iterative deconvolution algorithm. The residual deviation from a delta function for PSFs obtained at different times, displayed as a map or converted to a single number by any of a variety of means (total power in the residual image, for example), could be used as a stability benchmark.

An advantage of a deconvolution-based metric is its close relevance to a very basic post-processing operation; it can potentially tell an observer how useful deconvolution will be and how far it can be taken. A disadvantage is that deconvolution can be a somewhat subjective exercise, since the resulting map, in the presence of noise, is never unique; obvious variables in standard iterative algorithms are the initial trial map and the number of iterations. 
We have experimented with the Maximum Entropy Method (MEM) ${ }^{9}$ and with a variant of the Richardson-Lucy $(\mathrm{RL})^{10,11}$ deconvolution algorithm; the results discussed here were obtained with MEM. Initial trial maps were consistently taken to be flat (constant) maps.

\subsection{Example: Deconvolution of the Entire PSF with Itself}

To illustrate deconvolution as a test of PSF stability, we return to the data set presented in Figure 3, a series of short exposure PSF images separated by roughly 2.5 seconds of time. We show in Figure 6 the results of deconvolving the first, second, and last (eighth) image in the series through a fixed, arbitrary number of iterations of the maximum entropy algorithm, using the first image as the formal PSF in each case. The gap in time from the first to the second of the images in Figure 3 was about 2.5 seconds, and from the first to the eighth about 18 seconds.
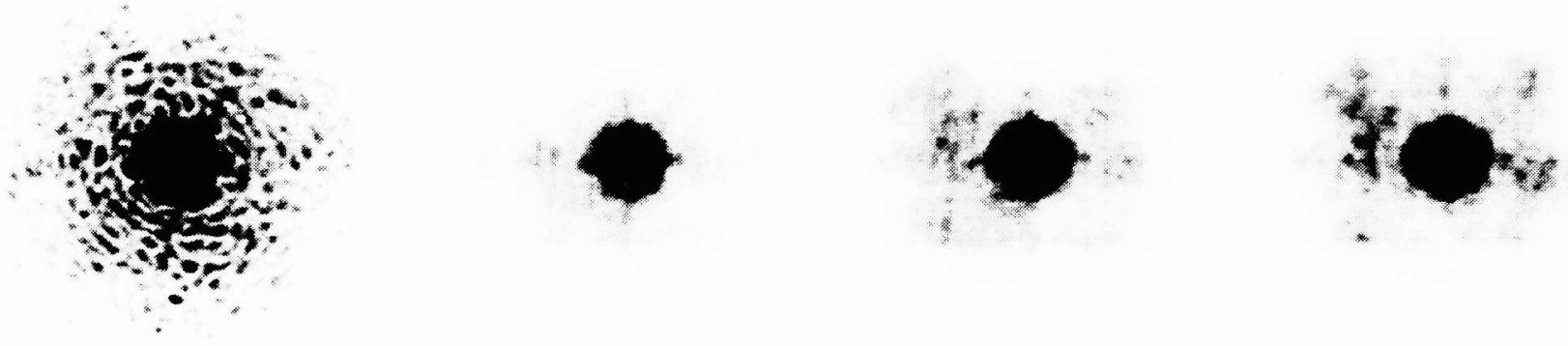

Figure 6. Whole-image deconvolution results, involving 1st, 2nd, and 8 th images in Figure 3, and 10 MEM iterations: (a) Fiducial PSF, the first image shown in Figure 3, with FWHM $=3.5$ pixels; (b) MEM result of 1st image deconvolved with itself, FWHM $=3.5$ pixels; (c) MEM result of 2nd image deconvolved with 1st, FWHM = 3.8 pixels; (d) MEM result of 8 th image deconvolved with 1st, FWHM $=5.9$ pixels. The gradual drift of the PSF with time is shown in the development of extended residual structure in the last three panels.

The increase in outlying structure in the third and fourth grayscales of Figure 6 compared to the second indicates that the PSF is changing noticeably with time. The symmetry of the deconvolved patterns in each of those cases suggests that a large part of the PSF drift in the halo is due to the growth of the waffle mode artifact. The larger width of the residual deconvolved peak indicates that component of the PSF is drifting as well; of course, deconvolution is insensitive to relative offsets (tip/tilt differences) among these short exposures.

\subsection{Deconvolution Metric Applied to the Main Peak of the PSF}

We have made preliminary investigations of deconvolution-based metrics applied to evaluating the time stability of the main lobe of the PSF. Before any processing, we first blanked the rest of the image to avoid distorting the results with asymmetries in the fainter Airy ring or halo; if deconvolution-based metrics of the sort contemplated here are applied to those fainter regions, it it even more important to blank the main peak, which would otherwise dominate the deconvolution. (This procedure has no theoretical basis if using deconvolution to recover an accurate underlying image, but we are instead using deconvolution to compare the structure of an arbitrarily isolated part of the PSF in two images).

We find that deconvolving one lobe with another or with an Airy pattern or Gaussian of comparable size leads to very smooth reduction of the deconvolved map's width with number of iterations. Further, the maps are largely circularly symmetric, indicating a high degree of similarity in the shape of the main lobe, as is indicated by inspection. These results can be quantified in a metric in a number of ways. The two-dimensional deconvolved map after a specified number of iterations may be used directly. Or, single-parameter metrics describing the gross degree of similarity may be computed from the average FWHM of the deconvolved peak. More detailed still, the azimuthal symmetry of the deconvolved peak, describing the similarity of the shapes of the images being compared, may be quantified by the ratio of major and minor axes of a best-fit Gaussian. It appears that lower-Strehl images have main peaks with shapes of noticeably lower quality in terms of their radial smoothness and azimuthal symmetry. 


\section{NEW METRICS: VARIANCE-MAP BASED}

\subsection{Variance-Map Metric Applied to the PSF Halo: Half-Minute Time Series}

Beyond the gross assessment of the total flux lost from the main peak of the PSF provided by the Strehl ratio, we would like for many applications to have a measure that characterizes the structure and stability of the halo in more detail. In particular, although any intensity diverted into the halo is undesirable, atmospheric or instrumental artifacts that persist for very short or very long time scales may be somewhat more benign in practical situations; further, as we have seen in the early sections of this paper, many instrumental artifacts may be identified by their spatial signature, possibly permitting correction. We would thus like to quantify the variation of intensity as a function of position in the PSF halo.

A straightforward metric providing this information for a time series of adaptively-corrected images is a "variance map", formed simply by computing standard statistics, pixel-by-pixel, on the photon counts (intensity) over the set of images. Regions of large variance are naturally less conducive to sensitive searches for faint companions, and fundamental limits to such searches may be identified. Experimentally-measured image variance may also be compared with the theoretical predictions for Poisson noise, which governs photon arrival statistics, and for "speckle noise", which should be dominant in the halo ${ }^{12}$.

The results of a preliminary variance-map study with a small time series of short-exposure PALAO images are shown in Figure 7 . The data set consisted of 120.2 -second images spaced by about 2.5 seconds of time. The pixelby-pixel average of photon counts is presented in Figure 7 (left); the variance over the 12 images of counts at each pixel is shown in Figure 7 (center). Finally, the speckle-noise-corrected variance is shown in Figure 7 (right): here, the expression for speckle noise derived by Racine et al. ${ }^{12}$ has been subtracted from the experimentally-determined variance map next to it, and the (inverted) result has been displayed on the same grayscale as the variance map.
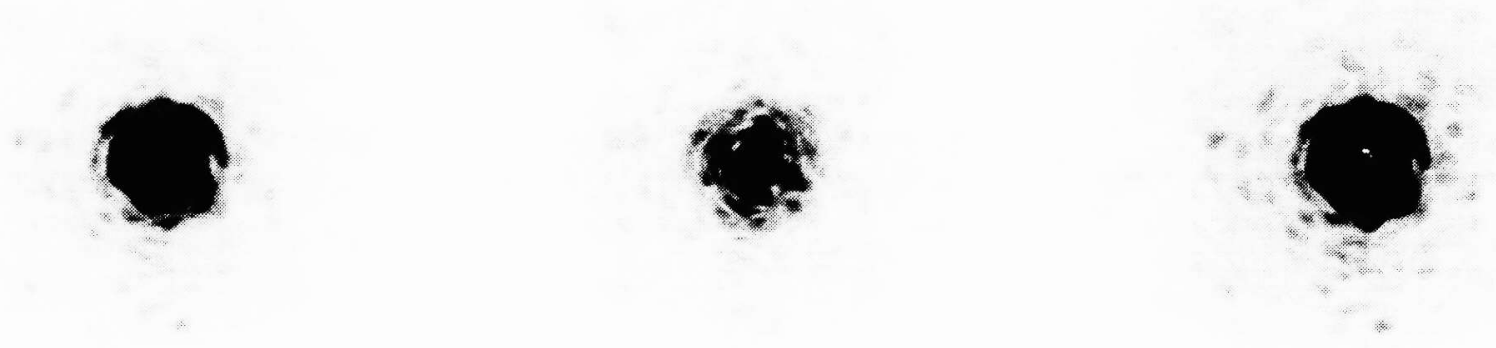

Figure 7. (Left) Average of 12 consecutive 0.2 -second-exposure images at $\lambda=2.2 \mu \mathrm{m}$, spanning about 30 seconds; (center) Pixel-by-pixel variance plot for that time series of images; (right) Speckle-noise-corrected variance map [NOTE: this is actually the inverse of that map, i.e., the value of speckle noise calculated from the average intensity map is generally larger than the observed variance].

The speckle-noise-corrected map may perhaps be somewhat smoother in some areas of the halo. It also shows numerous bright features surviving or even enhanced by the noise subtraction. This behavior might be expected from ghosts or other artifacts that remain fixed in position, and don't wander as speckles would. Their fluctuations are then given by Poisson statistics, and any over-correction based on the much larger fluctuations that would be predicted from speckle noise would be noticeable in a map like the right panel of Figure 7. A similar argument would apply to any bright knots on high-order Airy rings, analogous to the ones studied in $\S 3$ and depicted in Figure 2 , if they shared the temporal persistence of those artifacts seen on the first Airy ring. It should be cautioned, though, that the small number of images in this preliminary study may be affecting the statistics here, and these questions bear further investigation. 


\section{PSF MODULATION}

An interesting operational issue related to the topic of this paper bears mention here: our early observations with PALAO confirm the utility of distinguishing faint companions from PSF artifacts by intentionally modulating the low-level structure of the PSF. We have already found a low-tech, low-bandwidth PSF modulation to be very useful: rotating the position angle of the instrument on the sky.

More ambitious schemes are easily automated into the procedure that fine-tunes the instrumental PSF by calibrating individual subaperture spot centroid offsets in the wavefront sensor. As discussed in $\S 3$, the closed-loop operation of the deformable mirror drives the wavefront to the shape that produces these requested offsets. The shape is normally chosen to give something approximating the classic Airy pattern, but arbitrary pupil-plane phase functions may be inserted, up to a degree of control limited by the number of actuators on the deformable mirror.

If an intentionally large component of coma, for example, were added to the PSF, the secondary maximum of the Airy pattern would be suppressed on one side at the expense of an enhancement on the other. This suppression of the PSF's extended structure could dramatically aid companion searches, and could be easily modulated for further discrimination of instrumental effects.

\section{ACKNOWLEDGMENTS}

We thank Robert Brucato for useful discussions on numerous aspects of this project, and Hal Petrie for insights into the support mechanism for the $5 \mathrm{~m}$ primary.

\section{REFERENCES}

1. M. Troy, R. Dekany, G. Brack, F. Shi, B. Oppenheimer, T. Trinh, E. Bloemhof, T. Kieu, T. Hayward, B. Brandl, and F. Dekens, "Palomar Adaptive Optics Project: Status and performance", in Adaptive Optical Systems Technology, F. J. Roddier, ed., Proc. SPIE 4007, 2000 (this volume).

2. R. G. Dekany, "The Palomar Adaptive Optics System", in Adaptive Optics, OSA Technical Digest Series (Optical Society of America, Washington, D.C.) Vol. 13, pp. 40-42, 1996.

3. B. R. Oppenheimer, "Direct Detection of Brown Dwarf Companions of Nearby Stars", Ph. D. dissertation, California Institute of Technology, 1999.

4. E. E. Bloemhof and R. G. Dekany, "Degradation of the Adaptive Optics Point-spread Function due to Noncommon-path Flexure: A Simple Laser Metrology Solution", in Astronomy with Adaptive Optics, Proc. of ESO/OSA Topical Meeting on Astronomy with Adaptive Optics, Sonthofen, FDR, D. Bonaccini, ed., ESO Conf. and Wkshop Proc. No. 56, pp. 481-489, 1999.

5. B. Brandl, T. L. Hayward, and J. R. Houck, "Camera Design Report: PHARO (Palomar's High Angular Resolution Observer)", Cornell University/PALAO Project Internal Report, September 20th, 1996.

6. R. Dekany, M. Troy, G. Brack, T. Trinh, F. Shi, D. Palmer, M. E. Brown, B. R. Oppenheimer, D. Banfield, T. Hayward, and B. Brandl, "Solar System Science with Subarcsecond Slit Spectroscopy", in Adaptive Optical Systems Technology, F. J. Roddier, ed., Proc. SPIE 4007, 2000 (this volume).

7. B. R. Oppenheimer, R. Dekany, T. Hayward, B. Brandl, and M. Troy, "Companion Detection Limits with Adaptive Optics Coronagraphy", in Adaptive Optical Systems Technology, F. J. Roddier, ed., Proc. SPIE 4007, 2000 (this volume).

8. J. A. Marshall, M. Troy, and R. Dekany, "Anisoplanicity Studies within NGC6871", in Adaptive Optical Systems Technology, F. J. Roddier, ed., Proc. SPIE 4007, 2000 (this volume).

9. J. M. Hollis, J. E. Dorband, and F. Yusef-Zedeh, "Comparing Restored HST and VLA Imagery of R Aquarii", Ap. J. 386, pp. 293-298, 1992.

10. W. H. Richardson, J. Opt. Soc. Am. 62, p. 55, 1972.

11. L. B. Lucy, "An Iterative Technique for the Rectification of Observed Distributions", A. J. 79, pp. 745-754, 1974.

12. R. Racine, G. A. H. Walker, D. Nadeau, R. Doyon, and C. Marois, "Speckle Noise and the Detection of Faint Companions", P.A.S.P 111, pp. 587-594, 1999. 